\title{
Frequency Stepped Pulse Train Modulated Wind Sensing Lidar
}

\section{Olesen, Anders Sig; Pedersen, Anders Tegtmeier; Rottwitt, Karsten}

\section{Published in:}

Proceedings of SPIE - The International Society for Optical Engineering

Link to article, DOI:

$10.1117 / 12.893585$

Publication date:

2011

Document Version

Publisher's PDF, also known as Version of record

Link back to DTU Orbit

Citation (APA):

Olesen, A. S., Pedersen, A. T., \& Rottwitt, K. (2011). Frequency Stepped Pulse Train Modulated Wind Sensing Lidar. Proceedings of SPIE - The International Society for Optical Engineering, 8159, 815900.

https://doi.org/10.1117/12.893585

\section{General rights}

Copyright and moral rights for the publications made accessible in the public portal are retained by the authors and/or other copyright owners and it is a condition of accessing publications that users recognise and abide by the legal requirements associated with these rights.

- Users may download and print one copy of any publication from the public portal for the purpose of private study or research.

- You may not further distribute the material or use it for any profit-making activity or commercial gain

- You may freely distribute the URL identifying the publication in the public portal 


\title{
Frequency Stepped Pulse Train Modulated Wind Sensing Lidar
}

\author{
Anders Sig Olesen, Anders Tegtmeier Pedersen and Karsten Rottwitt \\ DTU Fotonik, Department of Photonics Engineering, Technical University of Denmark, \\ Ørsteds Plads 343, 2800 Kgs. Lyngby, Denmark;
}

\begin{abstract}
In this paper a wind sensing lidar utilizing a Frequency Stepped Pulse Train (FSPT) is demonstrated. One of the advantages in the FSTP lidar is that it enables direct measurement of wind speed as a function of distance from the lidar. Theoretically the FSPT lidar continuously produces measurements as is the case with a CW lidar, but at the same time with a spatial resolution, and without the range ambiguity originating from e.g. clouds. The FSPT lidar utilizes a frequency sweeping source for generation of the FSPT. The source generates a pulse train where each pulse has an optical carrier frequency shifted a set quantity relative to the carrier frequency of the previous pulse. In the scheme presented here, the measured frequency depends on the distance from which the signal originates. The measured frequency is related to the Doppler frequency shift induced by the wind and an integer number of frequency shifts corresponding to a specific distance. The spatial resolution depends on the repetition rate of the pulses in the pulse train. Directional wind measurements are shown and compared to a CW lidar measurement. The carrier to noise ratio of the FSPT lidar compared to a CW lidar is discussed as well as the fundamental differences between the two systems. In the discussion we describe the most dominant noise sources in our system and what influences these have on the FSPT lidar's ability to measure under different scattering conditions.
\end{abstract}

Keywords: Laser anemometer, coherent lidar, fiber laser, range unambiguity, frequency modulation

\section{INTRODUCTION}

The Frequency Stepped Pulse Train (FSPT) modulated lidar has previously been suggested as a means to achieve range resolved measurements of atmospheric wind speeds while maintaining a high duty cycle compared to other pulsed lidar systems. Earlier measurements have demonstrated the advantages of the technique on a moving hard target. ${ }^{1}$ In this paper we show range resolved measurements of actual atmospheric wind speed performed with this technique and discuss the Carrier to Noise Ratio (CNR) of the FSPT modulated lidar compared to $\mathrm{CW}$ and pulsed lidars.

A FSPT consists of a sequence of laser pulses each temporally and spectrally separated from each other by a fixed time and frequency. This separation allows for an unambiguous mapping of spatial range cells into well defined slots in the measured spectrum. The temporal separation should be kept as small as possible to ensure a quasi-CW optical output and thereby a high duty cycle. On one hand the spectral separation should be larger than the highest Doppler shift to be measured to avoid range ambiguities, while on the other hand it must be small enough to be within the detector bandwidth.

This paper explains the basic concept of the FSPT modulated lidar as well as a method for generating a suitable train of pulses. Finally range resolved wind speed measurements produced by a FSPT modulated lidar are presented and compared to CW lidar measurements.

Further author information: (Send correspondence to A.S.O.)

A.S.O.: E-mail: aole@fotonik.dtu.dk, Telephone: +45 45256879

A.T.P.: E-mail: antp@fotonik.dtu.dk, Telephone: +45 45256357

K.R.: E-mail: karo@fotonik.dtu.dk, Telephone: +45 45256384

Lidar Remote Sensing for Environmental Monitoring XII, edited by Upendra N. Singh,

Proc. of SPIE Vol. 8159, 81590O - ( ) 2011 SPIE · CCC code: 0277-786X/11/\$18 - doi: 10.1117/12.893585

Proc. of SPIE Vol. 8159 815900-1 


\section{METHOD}

The FSPT modulated lidar emits trains of optical pulses where each pulse in a train is equidistantly shifted in frequency by $\Delta f$ compared to the pulse prior to itself and thereby a discrete linear frequency sweep is obtained. The concept behind the FSPT modulated lidar is illustrated in Figure 1. Each pulse in the FSPT has a pulse duration of $T_{\text {pulse }}$ and the inter-pulse duration is given by $T_{\text {inter }}$. A Local Oscillator (LO) is generated as a copy of the transmitted FSPT. In this configuration the beating between the received scattered light and the LO generates peaks in separate frequency slots as shown in the bottom panel of Figure 1. These frequency slots are defined by $\Delta f$ through

$$
\begin{aligned}
& f_{i}=\left(i-\frac{3}{2}\right) \Delta f, \\
& f_{i}^{\prime}=\left(i-\frac{1}{2}\right) \Delta f,
\end{aligned}
$$

where the $i^{\text {th }}$ slot stretches from $f_{i}$ to $f_{i}^{\prime}, i$ being an integer number starting from 1 . Spatially each frequency slot corresponds to a given range cell because of the delay between light scattered in the atmosphere and the $\mathrm{LO}$ as shown in the top and middle panel of Figure 1. This means that light scattered, e.g. in the second range cell will beat against an LO that is shifted by $\Delta f$ compared to the transmitted frequency, and by making sure $\Delta f$ is larger than the induced Doppler shift, $f_{D}$, a unique mapping of the range cell into the frequency slot is achieved. The $i^{\text {th }}$ range cell extending from $x_{i}$ to $x_{i}^{\prime}$ is described by

$$
\begin{gathered}
x_{i}=\left[(i-2) T_{\text {pulse }}+(i-1) T_{\text {inter }}\right] \frac{c}{2}, \\
x_{i}^{\prime}=\left[i T_{\text {pulse }}+(i-1) T_{\text {inter }}\right] \frac{c}{2},
\end{gathered}
$$

where $\mathrm{c}$ is the speed of light.

As shown in Figure 1 range cells partly overlap if $T_{\text {pulse }}>T_{\text {inter }}$, and the centre of the first range cell will be situated at a distance of $0 \mathrm{~m}$. The range cell positions can be changed by introducing a time delay to the LO and if sufficiently large the cropping of the first range cell is avoided. ${ }^{1}$ The wind signal frequency generated in the $i^{\text {th }}$ range cell is defined by

$$
f_{\text {wind }, i}=f_{D}\left(x_{i}: x_{i}^{\prime}\right)+(i-1) \Delta f,
$$

and the line-of-sight speed in range cell $i$ is thereby given as

$$
V_{L O S, i}=\frac{\lambda}{2}\left(f_{w i n d, i}-(i-1) \Delta f\right) .
$$

In this configuration the first frequency slot lacks the capability of determining the sign on the measured velocity. This can be seen from Eqs. (1) and (2) which calculates to a frequency slot from $-\Delta f / 2$ to $\Delta f / 2$ for the first frequency slot. The firsts frequency slot therefore only spans from 0 to $\Delta f / 2$ with positive and negative frequencies being indistinguishable. The directional ambiguity may therefore be avoided if a frequency offset is introduced to the LO. The offset should be larger than $\Delta f / 2$ to avoid any velocity ambiguities.

It is noted that the FSPT should contain enough pulses such that the received backscattered light from the last pulses in a FSPT beating with the first pulses from the subsequent LO FSPT generates frequencies outside the measured frequency range to avoid any ambiguities. Note that from the $n^{\text {th }}$ LO pulse only $n$ range cells contribute to the measurement. By using a FSPT consisting of several pulses the lack of signal from higher order range cells in the initial pulses is neglected.

\section{FSPT MODULATED LIDAR SYSTEM DESIGN}

A focused CW lidar system based on an all fiber setup has been modified for the use as a FSPT modulated lidar. The layout of the system used is shown in Figure 2. As the FSPT generator a Lightwave Synthesized Frequency Sweeper (LSFS) ${ }^{2,3}$ is used. Due to the low power output of this the FSPT is amplified by an external Erbium Doped Fiber Amplifier (EDFA) to an average power of $29 \mathrm{dBm}$. The amplified FSPT is coupled into the 

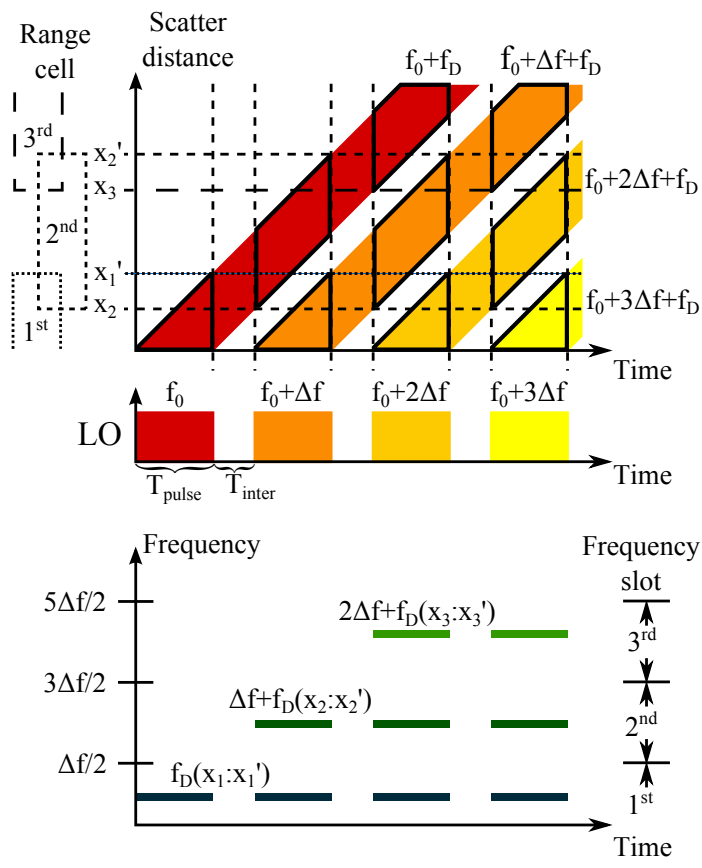

Figure 1. Top panel: Time-space representation of the received scatter from an FSPT modulated lidar. Range cells are defined by the interaction time between pulses of the LO and scattered pulses governed by the pulse duration $T_{p u l s e}$ and inter-pulse duration $T_{\text {inter }}$. Middle panel: Representation of LO in the same time frame as the time-space representation. Bottom panel: Time-frequency representation of the heterodyne signal generated by a photo detector. The frequency slot $i$ contains Doppler shifted frequencies from range cell $i$ plus a frequency shift $(i-1) \Delta f$.

telescope through a circulator and focused into the atmosphere. The telescope is build with a lens diameter of 7 $\mathrm{cm}$, and an adjustable focus. A reflection from the end facet of the delivery fiber generates the non delayed, non offset LO. The backscattered light is collected through the telescope and together with the LO mixed onto an AC coupled photodiode via the circulator. The heterodyne signal generated by the photo detector is filtered by a passive low pass filter and a passive high pass filter to remove the pulse shapes and avoid aliasing. The filtered signal is then sampled at $400 \mathrm{MHz}$ by a digitizer and digitally processed in a computer. The pulse shapes are filtered to avoid frequency components from the pulse shapes and to reduce the voltage span of the signal and thereby decrease the quantization error.

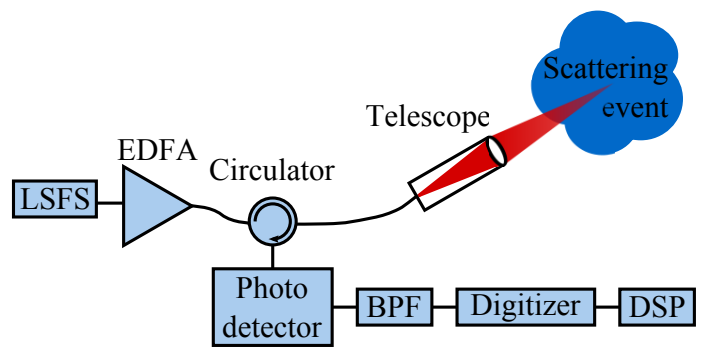

Figure 2. Illustration of the FSPT modulated lidar. The FSPT is generated by the LSFS and amplified by the EDFA. The amplified FSPT is sent into a telescope through a circulator and transmitted into the atmosphere. A weak reflection at the circulators fiber end facet simultaneously generates the LO. The backscattered light is received by the same telescope and transmitted together with the LO to a photo detector through the circulator. The heterodyne signal from the photo detector is filtered by a Band Pass Filter (BPF) to remove pulse shapes and avoid aliasing before it is sampled by a digitizer and processed by a Digital Signal Processor (DSP). 
Sections of the sampled signal corresponding to the top of each LO pulses are cut out and Fourier transformed to generate a spectrum. These spectra are then averaged to form an averaged signal spectrum. A similar measurement is performed with a shutter in front of the telescope to exclude the signal and measure the noise spectrum of the system. To obtain the CNR spectrum the averaged signal spectrum is divided by the averaged noise spectrum.

\subsection{Lightwave Synthesized Frequency Sweeper}

The configuration of the LSFS used as the FSPT generator is illustrated in Figure 3. The loop is seeded by a $7 \mathrm{~mW}$ fiber laser at a wavelength of $1548 \mathrm{~nm}$. One seed pulse for every FSPT is generated by an acousto-optic modulator $(\mathrm{AOM})$ with an extinction ratio of $63 \mathrm{~dB}$. In addition to generation of the seed pulse, the AOM induces a frequency shift of $40 \mathrm{MHz}$ to the carrier frequency of the light. The seed pulse is coupled into the loop through a 50/50 coupler and from there into an EDFA of which the gain is set to match the total attenuation of the loop. From the EDFA the pulse then passes through a Band Pass Filter (BPF), another AOM which induces a new frequency shift of $40 \mathrm{MHz}$, a single mode transmission fiber, a polarization controller, and back into the 50/50 coupler where half the pulse power is coupled out of the ring and the other half back into the ring. By repeating this circulation through the loop a new pulse is generated each time with a frequency shifted by $\Delta f$ $=40 \mathrm{MHz}$ compared to the previous pulse and the FSPT is thereby generated.

The BPF is used to reduce the ASE noise generated by the EDFA, ${ }^{4}$ and the polarization controller is used to compensate for the polarization change throughout the ring. A polarizer at the output of the LSFS ensures that all pulses in the output FSPT has the same polarization which is desirable when used for heterodyne detection.

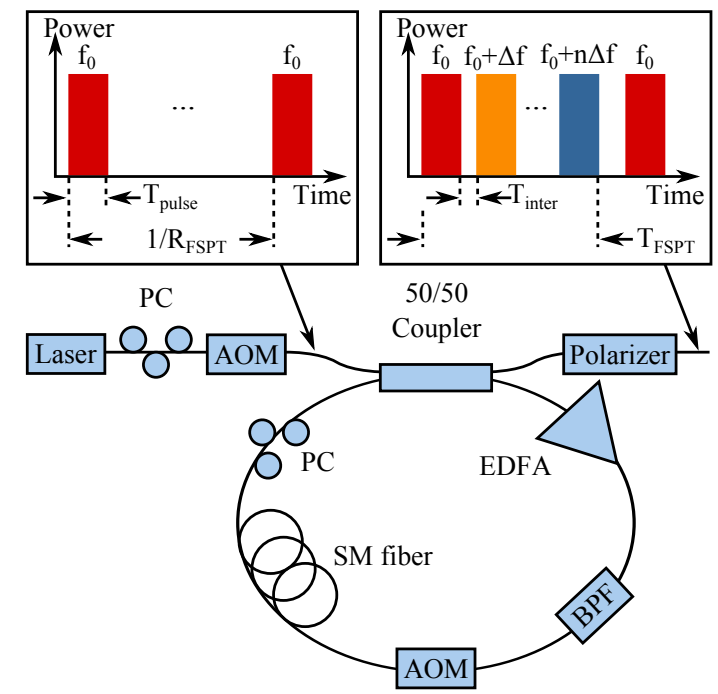

Figure 3. Illustration of the LSFS setup. A seed pulse of duration $T_{\text {pulse }}$ is generated by modulation of the cw laser light by the seed AOM at a rate $R_{F S P T}$. Half of the seed pulse is coupled into the loop by a $50 / 50$ coupler and half is coupled out of the LSFS through the polarizer as the first pulse in the FSPT. The pulse then circulates the ring and each time generates the next pulse in the FSPT delayed by $T_{\text {inter }}$ compared to the previous pulse and frequency shifted $\Delta f$. The loop AOM induces the frequency shift as well as controls the FSPT duration $T_{F S P T}$. The EDFA compensates for losses in the loop and the BPF minimizes noise growth. The pulse to pulse time $T_{p u l s e}+T_{\text {inter }}$ is governed by the loop length adjustable by the length of the Single Mode (SM) transmission fiber. The Polarization Controller (PC) is used to compensate for the polarization change through the ring and the polarizer is ensuring a constant output polarization for all pulses.

In this configuration the seed AOM controls the pulse duration $T_{\text {pulse }}$ and the repetition rate of the FSPT $R_{F S P T}$. The loop length, which is adjustable through the length of the transmission fiber, defines the pulse to pulse duration $T_{\text {pulse }}+T_{\text {inter }}$, and the loop AOM governs the FSPT duration $T_{F S P T}$. $T_{F S P T}$ is set as high as possible such that as short a down time between the FSPTs as possible is achieved, but no higher than leakage between the FSPTs is avoided. 


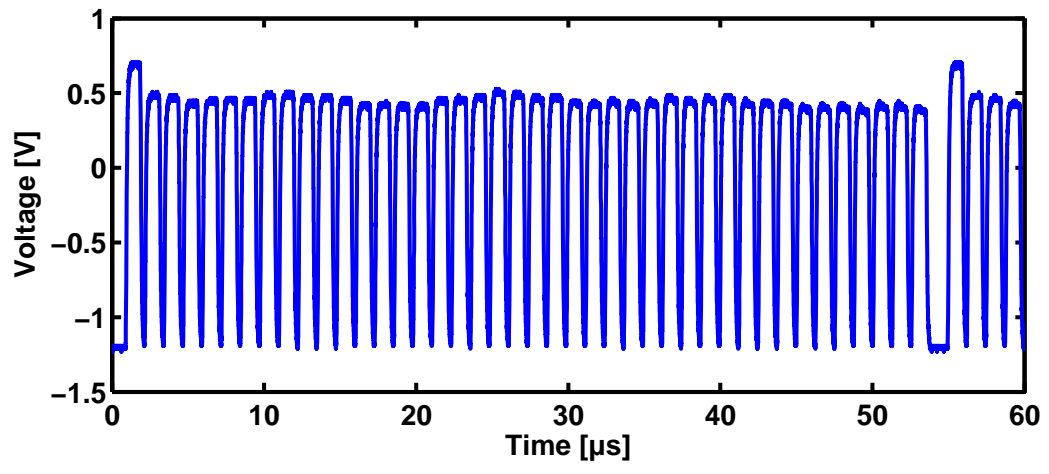

Figure 4. FSPT generated by the LSFS with $R_{F S P T}=18.5 k H z, T_{F S P T}=53 \mu s, T_{\text {pulse }}=1 \mu s, T_{\text {inter }}=0.26 \mu s$ and a frequency shift $\Delta \mathrm{f}$ of $40 \mathrm{MHz}$

Figure 4 shows a FSPT generated by the LSFS with $R_{F S P T}=18.5 \mathrm{kHz}, T_{F S P T}=53 \mu \mathrm{s}, T_{p u l s e}=1 \mu \mathrm{s}$, $T_{\text {inter }}=0.26 \mu \mathrm{s}$ and a frequency shift $\Delta f$ of $40 \mathrm{MHz}$. In this configuration each FSPT contains 42 pulses and with an average power of $29 \mathrm{dBm}$ this amounts to a pulse energy of $\sim 1 \mu \mathrm{J}$ transmitted into the atmosphere. A square pulse shape is used to produce a constant LO power when cutting the pulses for processing and thereby increasing the time over which each spectrum is measured. With this configuration line of sight wind speeds of up to $15 \mathrm{~m} / \mathrm{s}$ are measurable without range ambiguity. For speeds above $15 \mathrm{~m} / \mathrm{s}$ the signal would appear in the neighboring frequency slot and hence appear to be measured in the neighboring range cell and with an opposite direction.

\section{MEASUREMENT RESULTS}

The wind speed measurements performed with the system described above are shown in Figure 5 . In these measurements each range cell extends over $300 \mathrm{~m}$ and, due to the low inter-pulse duration compared to the pulse duration, a large overlap between consecutive range cells occurs. The range cells span from 0 - 150 m, 39 - 339 m, $228-528 \mathrm{~m}$ and $417-717 \mathrm{~m}$ for the first, second, third and fourth range cell respectively. The corresponding frequency slots extends from 0 - $20 \mathrm{MHz}, 20-60 \mathrm{MHz}, 60-100 \mathrm{MHz}$ and 100 - $140 \mathrm{MHz}$, respectively. To illustrate the directional dependence of the wind speed the two measurements shown were taken in different directions, one in the general wind direction and the other up against the wind direction. Both of them were taken with a focus distance set to $350 \mathrm{~m}$ and show measurements of wind speeds in both second and third range cell. The measurements in Figure 5 were achieved in high-scattering conditions where the aerosol density was high. The peak frequencies are 50.2 MHz and 90.2 Mhz for the measurement in the wind direction and 31.37 $\mathrm{MHz}$ and $71.37 \mathrm{MHz}$ for the measurement against the wind direction. According to Equation 6 this corresponds to a line of sight wind speeds of $7.9 \mathrm{~m} / \mathrm{s}$ in the wind direction and $6.7 \mathrm{~m} / \mathrm{s}$ against the wind direction. With a focus at $350 \mathrm{~m}$ the return signal is manly expected to originate from the overlapping sections of the second and third range cell and identical line of sight wind speed in the two range cells are therefore as expected. The difference in line of sight wind speeds between the two measurements originates from changing wind conditions and that they are not measuring the wind speed in the exact opposite directions of each other.

For comparison a measurement using a CW source instead of the FSPT source is shown in Figure 6. The CW lidar in this case uses the same setup as the FSPT lidar and transmits the same average power, only the FSPT source was changed to a CW laser source. The CW laser source in this case was the CW laser source used in the LSFS setup. By using the same laser source in the CW lidar as in the FSPT lidar the measurements only differs in the method used and a qualitative comparison of the methods can be made. The measurement was performed under the same high-scattering conditions as the FSPT lidar measurements. The line of sight wind speed measured by the CW lidar is $4.5 \mathrm{~m} / \mathrm{s}$ which is a lower than the FSPT lidar measurement but this could be expected due to changing wind conditions between the measurements.

When comparing the CW lidar measurement and the FSPT lidar measurement it is seen that the CNR of the CW lidar is approximately a factor of $\sim 14$ times higher than the FSPT lidar. For the FSPT lidar to be able 

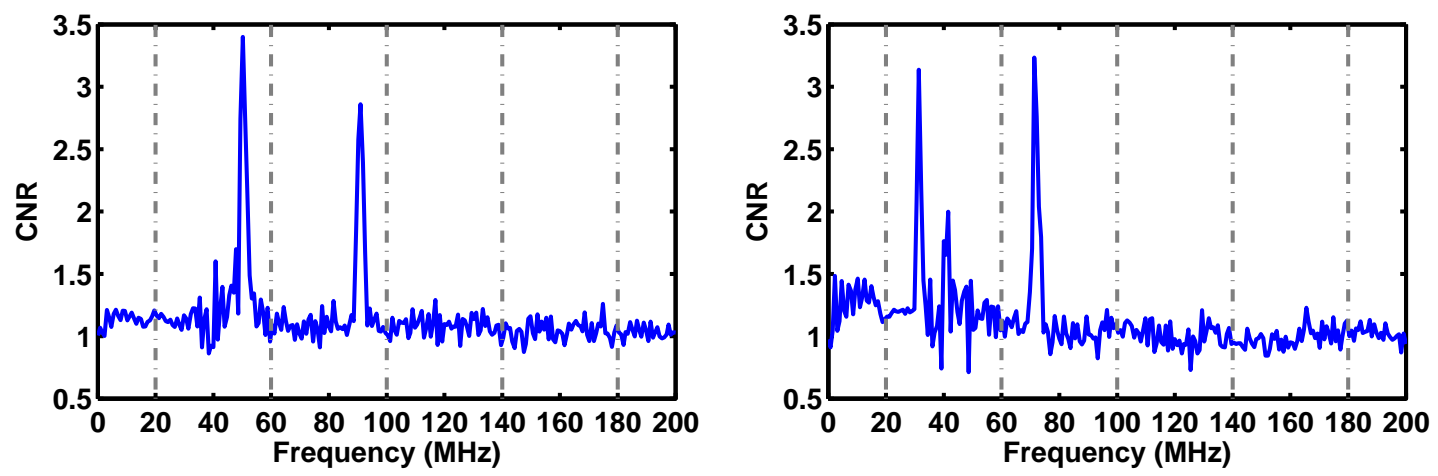

Figure 5. Spectral measurements of atmospheric wind speed by the FSPT modulated lidar at a telescope focus length of $350 \mathrm{~m}$ with and against the wind direction, left and right respectively. Measurements are performed with the FSPT settings: $R_{F S P T}=22.5 \mathrm{kHz}, T_{F S P T}=50 \mu \mathrm{s}, T_{\text {pulse }}=1 \mu \mathrm{s}, T_{\text {inter }}=0.26 \mu \mathrm{s}, \Delta \mathrm{f}$ of $40 \mathrm{MHz}$, and average power of 29 $\mathrm{dBm}$. Each spectrum is an average over 3894 spectra each calculated from each their pulse and then divided by an equally calculated measurement of the noise spectrum. The dashed lines indicates the separation between the frequency slots.

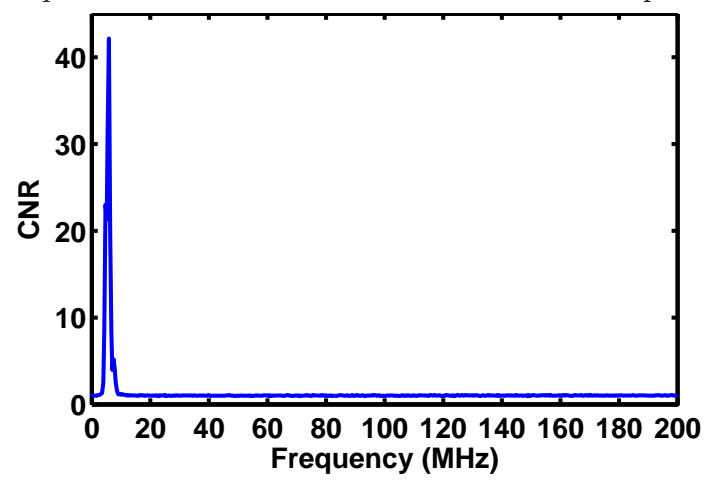

Figure 6. Spectral measurements of atmospheric wind speed by a CW lidar at a telescope focus length of $350 \mathrm{~m}$. Measurements performed with an average power of $29 \mathrm{dBm}$. The spectrum is an average over 2047 individual spectra divided by an equally averaged noise spectrum.

to measure under all atmospheric conditions it needs to have a CNR nearly as high as the CW lidar and noise differences between the two systems is therefore discussed in the following. The measurements were performed in a non homogeneous atmosphere and it is therefore not possible to directly compare the measurements. A more rigorous study should be made under more steady conditions.

The highest possible CNR is achieved when the noise in the system is dominated by the shot noise. Figure 7 shows a measurement of the noise level of the FSPT lidar and the CW lidar relative to the dark noise of the systems. The three largest contributions to noise in the CW lidar are dark noise, from detector and electronics, Relative Intensity Noise (RIN), from fluctuations in laser power, and shot noise. When dark noise is dominant the noise level is independent of the optical power at the detector, whereas when RIN is dominant the noise level increases with the square of the power. Only when the noise is dominated by shot noise does it have linear dependence of power. ${ }^{5}$ Since the carrier strength also increases linear with power no further increase in CNR is achieved by increasing the power when operating the lidar in the shot noise limited regime. ${ }^{6}$ The noise level of the CW lidar shown in Figure 7 is growing linearly to at maximum of $3.5 \mathrm{~dB}$ higher than the dark noise level before saturating the detector. The dominant noise term in the $\mathrm{CW}$ lidar measurements was therefore the shot noise and an optimal CNR was achieved.

The noise level of the FSPT lidar would follow that of the CW lidar if no additional noise had been growing in the LSFS and only the center of the square pulses were used for the spectrum calculations. This is not the case. The noise level of the FSPT lidar is 7-12 dB higher than that of the CW lidar and does not grow linearly. This indicates that other noise terms contribute more than the shot noise and a lower CNR, as seen when comparing 


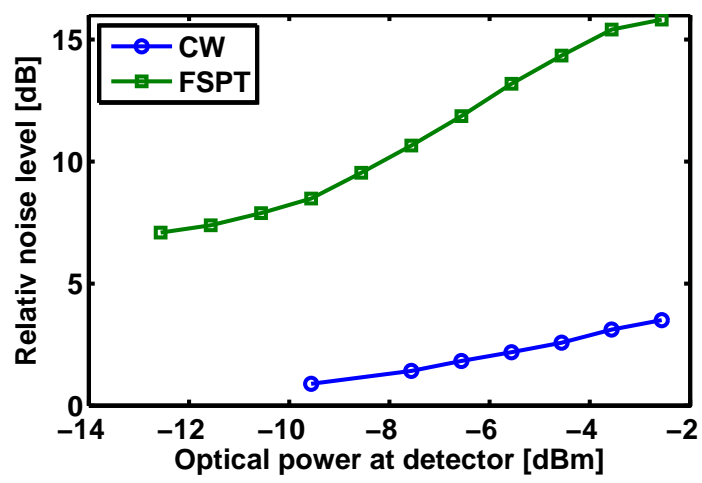

Figure 7. Noise level relative to dark noise of the system depending on the LO power on the detector for FSPT and CW lidar. The difference between the LO optical power at the detector and the output power of the EDFA is $32.56 \mathrm{~dB}$. The Saturation power of the photodetector is $-2.1 \mathrm{dBm}$.

Figure 5 and 6 , is therefore to be expected. One of the main sources to this increased noise has been seen to be the square pulse shapes which after filtering generates ringing at the front and back of each pulse which contributes to the noise level. This has been minimized by cutting shorter time sections from the center of the pulses when calculating the spectrums, but improvements are still possible. Other noise terms could originate from noise generated in the LSFS ring by the EDFA or leakage through the AOM.

Even if all noise sources are removed the fundamental difference between FSPT lidar and CW lidar gives rise to a lower CNR for the FSPT lidar. Theoretically the FSPT lidar is treated as a pulsed system where each individual pulse spectrum only experience return from one pulse in each range cell. In this configuration the power of the FSPT lidar is split out into multiple range cells and a lower CNR is therefore expected for the same average power. ${ }^{7}$

The fluctuations in the noise floor of the FSPT lidar seen in Figure 5 mainly originate from time dependent fluctuations in the pulse shapes. The pulses are not entirely identical as seen in Figure 4 and the overall FSPT shape varies slowly with time. Higher amount of averaging can compensate for the fluctuations and variations in pulse shapes but for the long term variations of the FSPT shape a more stable LSFS is needed. Ideally as low a number of averages as possible should be used since a high measuring rate is one of the merits of the FSPT compared to other pulsed lidar systems. The fluctuations should therefore primarily be reduced by reducing fluctuations in the FSPT which in theory also should reduce the noise and thereby increase the CNR.

\section{CONCLUSION}

We have described a FSPT modulated lidar and its merits. As a proof of concept we have shown measurements of wind speeds under high scatter conditions and proven that the FSPT lidar is capable of measuring line of sight wind speeds with directionality. In our configuration and under high scattering conditions a CNR close to 3 was achieved which is approximately 14 times lower than what is achieved with a CW lidar under same conditions. From the comparison between the FSPT lidar and a CW lidar it is found that a higher CNR is needed for the FSPT lidar to work under all atmospheric conditions.

\section{ACKNOWLEDGMENTS}

The Danish Agency for Science -Technology and Innovation, Research Infrastructure 2009, Grant No. 2136-080022 is thanked for financial support.

\section{REFERENCES}

1. Lindelöw, P., "Velocity sensing FSPT-modulated coherent lidar," Proc. 14th Coherent Laser Radar Conference, (2007). 
2. Hodgkinson, T. G. and Copping, P., "Pulsed operation of an optical feedback frequency-synthesizer," Electron. Lett. 26(15), 1155-1157 (1990).

3. Shimizu, K., Horiguchi, T., and Koyamada, Y., "Frequency translation of light waves by propagation around an optical ring circuit containing a frequency shifter. i. experiment," Appl. Opt. 32(33), 6718-6726 (1993).

4. Takano, K., Nakagawa, K., and Ito, H., "Influence of optical filters on pulse circulation fiber rings with a frequency shifter and EDFA," Opt. Express 14(22), 10313-10323 (2006).

5. Agrawal, G. P., [Fiber-Optic Communication Systems], Wiley-Interscience, New York (2002).

6. Sonnenschein, C. M. and Horrigan, F. A., "Signal-to-noise relationships for coaxial systems that heterodyne backscatter from the atmosphere," Appl. Opt. 10(7), 1600-1604 (1971).

7. Lindelöw, P. and Mohr, J. J., "Coherent lidar modulated with frequency stepped pulse trains for unambiguous high duty cycle range and velocity sensing in the atmosphere," in [Geoscience and Remote Sensing Symposium, 200\%. IGARSS 200\%. IEEE International], 2787-2790 (2007). 